\title{
A FUGACITY-BASED INDOOR RESIDENTIAL PESTICIDE FATE MODEL
}

\author{
DH Bennett ${ }^{1 *}$, E J Furtaw, $\mathrm{Jr}^{2}$ and TE McKone $\mathrm{C}^{3,4}$ \\ ${ }^{1}$ School of Public Health, Harvard University, Boston, MA, USA. \\ ${ }^{2}$ U.S. Environmental Protection Agency, Las Vegas, NV, USA \\ ${ }^{3}$ School of Public Health, University of California, Berkeley, CA, USA \\ ${ }^{4}$ Indoor Environment Dept., Lawrence Berkeley National Laboratory, Berkeley, CA, USA
}

\begin{abstract}
Dermal and non-dietary pathways are potentially significant exposure pathways to pesticides used in residences. Exposure pathways include dermal contact with residues on surfaces, ingestion from hand- and object-to-mouth activities, and absorption of pesticides into food. A limited amount of data has been collected on pesticide concentrations in various residential compartments following an application. But models are needed to interpret this data and make predictions about other pesticides based on chemical properties. In this paper, we propose a mass-balance compartment model based on fugacity principles. We include air (both gas phase and aerosols), carpet, smooth flooring, and walls as model compartments. Pesticide concentrations on furniture and toys, and in food, are being added to the model as data becomes available. We determine the compartmental fugacity capacity and mass transfer-rate coefficient for wallboard as an example. We also present the framework and equations needed for a dynamic mass-balance model.
\end{abstract}

\section{INDEX TERMS}

Pesticides, Exsure, Rsidential environments, Srfaces, Wallboard

\section{INTRODUCTION}

Screening level assessments have indicated that dermal and non-dietary pathways are potentially significant exposure pathways to pesticides used in homes, especially during the first few days following an application (Gordon et al. 1999; Zartarian et al. 2000). These pathways include dermal contact with treated surfaces by hands as well as other exposed skin, ingestion from both hand to mouth activities and contact with toys and other items that accumulate pesticide, and adsorption of pesticides into food, both from air-to-food transfers and from contact of the food with pesticide residue on surfaces (Gurunathan et al. 1998).

Among the many parameters needed to characterize exposure to pesticide residues in homes, the time history of surface concentrations, or loadings, is vital. Some data have been gathered on pesticide concentrations in homes following application, but only for a limited numbed of pesticides (Leidy et al. 1993; Byrne et al. 1998; Lewis et al. 2001). But there is constant change in the pesticides being used in residences as new pesticides are developed and as regulations change. This creates a demand for models that can be used to predict the time history and fate of a broad range of pesticides based on their chemical properties. To date, attempts to develop models to predict chemical behavior in indoor environments (Mackay et al. 1983; Neretnieks et al. 1993; Matoba et al. 1998), have been limited by insufficient data to calibrate the model parameters.

\footnotetext{
* Contact author email: dbennett@hsph.harvard.edu
} 
In this paper, we present the framework for a fugacity-based model of the residential environment and discuss the strategy for parameterizing such a model. Although a number of recent and ongoing studies are shedding more light on chemical partitioning to indoor surfaces, more data are still needed to fully parameterize such models. We present a list of current data needs.

\section{METHODS}

The work to develop and employ a fugacity model for residential environment includes several stages. First is the development of the framework. Key to model development are an approach for dealing with the movement of air and aerosols and the treatment of surfaces.

\section{Model Framework}

The framework for the fugacity based indoor residential model is flexible so we can include a variety of compartments. In Figure 1, we provide an example application with a treated room and five surface compartments. Compartments for the carpet and vinyl flooring that are treated with pesticide are included, as are three non-treated surface compartments that can act as potential reservoirs for the chemical; carpet, vinyl floor, and walls/ceiling. Our model framework can also accommodate other surface types, such as hard and soft sided furniture, as well as additional treated and untreated rooms. The air compartment connects all of the surfaces and removes chemical from the room through ventilation. However, the total number of compartment is limited by our ability to parameterize these compartments.

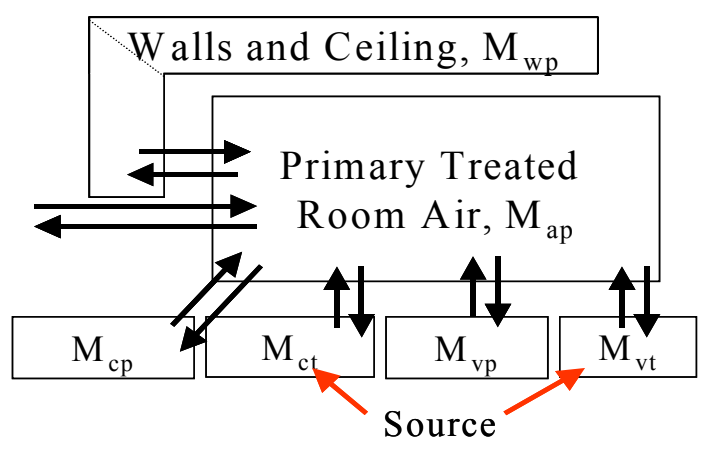

Figure 1. odel framework for indoor fugacity model

We use a mass transfer model, where the mass in each compartment is the state variable and the mass transfer between the compartments is defined by a transfer rate that accounts for both diffusive and advective transfers. Mass balance is defined by a set of differential equations that account for all gain and loss processes. The equation that accounts for the mass in the air of the primary treated room is:

$$
\frac{d M_{a p}}{d t}=-L_{a p} M_{a p}+T_{c a} M_{c t}+T_{v a} M_{v t}+T_{c a} M_{c p}+T_{v a} M_{v p}+T_{w a} M_{w p}
$$

where M's represent the compartment masses (in grams), T's are transfer factors (1/day), and $L_{a p}$ is a loss rate constant. We use $M_{a p}$ for mass in air, $M_{c t}$ for treated carpet, $M_{v t}$ for treated vinyl, $M_{\mathrm{cp}}$ for untreated carpet, $\mathrm{M}_{\mathrm{vp}}$ for untreated vinyl, $\mathrm{M}_{\mathrm{hp}}$ for untreated hardwood, $\mathrm{M}_{\mathrm{wp}}$ for walls and ceiling. We use $\mathrm{T}_{\mathrm{ca}}$ for transfer from the carpet to air, $\mathrm{T}_{\mathrm{va}}$ for vinyl to air, and $\mathrm{T}_{\mathrm{wa}}$ for walls to air. The loss rate constant from the air in each room is the sum of all loss and transfer processes:

$$
L_{a p}=k_{a}+T_{a p_{-} o u t}+T_{a p_{-} c t}+T_{a p_{-} v t}+T_{a p_{-} c p}+T_{a p_{-} v p}+T_{a p_{-} w p}
$$


where $\mathrm{k}_{\mathrm{a}}$ is the degradation rate in air and the other transfer factors are $\mathrm{T}_{\mathrm{ap} \_ \text {out }}$ for air to outdoors, $\mathrm{T}_{\mathrm{ap} \_ \text {ct }}$ for air to treated carpet, $\mathrm{T}_{\mathrm{ap} \_\mathrm{vt}}$ for air to treated vinyl, $\mathrm{T}_{\mathrm{ap} \_\mathrm{cp}}$ for air to untreated carpet, $\mathrm{T}_{\mathrm{ap} \_\mathrm{vp}}$ for air to untreated vinyl, and $\mathrm{T}_{\mathrm{ap} \_ \text {wp }}$ for air to walls.

Similar to Equation (1) there is a differential equation describing the mass in each of the surfaces as well. As an example, the equation for the treated carpet is defined below. In each case, $\mathrm{k}_{\mathrm{i}}$ is the transformation rate of the chemical, where the subscript $\mathrm{i}$ refers to the compartment type. Additionally, $\mathrm{S}_{\mathrm{i}}$ (mols/day) is the source rate of the chemical, where the subscript i refers to the compartment.

$$
\text { Treated Carpet: } \frac{d M_{c t}}{d t}=-L_{c} M_{c t}+S_{c t}+T_{a p_{-} c t} M_{a p} ; \quad L_{c}=k_{c}+T_{c a}
$$

\section{Movement of air and aerosols}

Movement of air and indoor aerosols to the outdoor environment occurs through ventilation. If a second or third room is considered, we use room to room air exchange. Both temporal and inter-home variability need to be considered when estimating the range of potential concentration values. Particle deposition and resuspension are important processes within the home. Rates vary by surface type, surface orientation, and particle size.

\section{Treatment of Surfaces}

Several researchers have made measurements that provide insight on the sink effect of surfaces in a room, including efforts to determine rates of sorption to and desorption from a surface. Some have developed isotherms to model the partitioning into a surface (Jorgensen et al. 2000). But it has not been established whether a chemical partitions primarily onto the surface of a material (Won et al. 2001), or whether the compounds diffuse into the pores and/or body of the material (Cox et al. 2001). Additionally, partitioning and diffusion may occur on different time scales, with short-term experiments accounting for partitioning onto the surface and long term experiments accounting for diffusion into the material. Presently, there are insufficient data to develop models that account for the diffusion into the materials. Another key issue is whether partitioning onto surfaces is a reversible or irreversible process. While most experiments have assumed reversibility, some compounds have been shown to have irreversible partitioning onto surfaces (Van Loy et al. 2001).

Jorgensen et al. proposed three general criteria for developing models of surfaces - (i) models should be based on sound physical/chemical description of the process (i.e., theoretically derived models are preferred over empirical models), (ii) models should be mathematically as simple as possible, and (iii) the model should "adequately" fit measured data (Jorgensen et al. 2000). In order to best comply with these criteria, we base our model on fugacity. Fugacity is a way of representing chemical activity at low concentrations and can be regarded physically as the partial pressure or escaping potential exerted by a chemical in one physical phase or compartment on another (Mackay 1991). At low concentrations, fugacity, $f(\mathrm{~Pa})$, is linearly related to concentration $\mathrm{C}\left(\mathrm{mol} / \mathrm{m}^{3}\right)$ through a fugacity capacity, $\mathrm{Z}\left(\mathrm{mol} / \mathrm{m}^{3}-\mathrm{Pa}\right), C=f Z$. The fugacity capacity defines the holding capacity of a material for a chemical substance based on the properties of both the material and the chemical. When two or more compartments are in equilibrium, the escaping tendency (the fugacity) of a chemical is the same in all phases. This characteristic of fugacity-based models often simplifies the mathematics involved in calculating partitioning. Fugacity models can also be used to represent a dynamic system in which the fugacities in two adjacent media are changing in time due to an imbalance of sources and losses (Mackay 1991). 


\section{RESULTS}

In order to illustrate the use of our model, we used available data for wallboard to define a fugacity capacity as well as the diffusive transfer rates between the air and the wallboard (Tichenor et al. 1991; Van Loy et al. 2001 ; Won et al. 2001). Much of the available experimental work provides information to establish sorption and desoprtion rates from a surface. From this work, we have determined that the associated fugacity capacity of the surface can be calculated as,

$$
Z_{\text {wallboard }}=\frac{k_{s} / k_{d}}{R T d_{w}}
$$

where $\mathrm{k}_{\mathrm{s}}$ is the sorption rate $(\mathrm{m} / \mathrm{h}), \mathrm{k}_{\mathrm{d}}$ the desorption rate $(1 / \mathrm{h}), \mathrm{R}$ the ideal gas constant $(\mathrm{Pa}-$ $\mathrm{m}^{3} / \mathrm{molK}$ ), $\mathrm{T}$ the ambient temperature (kelvins $[\mathrm{K}]$ ), and $\mathrm{d}_{\mathrm{w}}$ the thickness of the wallboard.

Fugacity capacities for various residential surfaces are calculated from partition coefficients, such as the air/surface equilibrium concentration ratio. Often these partition coefficients correlate with chemical properties such as vapor pressure or the octanol-air partition coefficient $\mathrm{K}_{\mathrm{oa}}$. To establish these relationships for painted wallboard, we consolidate data from all available sources (the data for nicotine from the Van Loy study was not included). This provides more data for assessing the correlation and allows us to compare the studies for consistency. For surface partition coefficients, we used both vapor pressure and $\mathrm{K}_{\mathrm{oa}}$, as predictors of surface partitioning. Figure 2 is a plot of the surface partition coefficient of wallboard versus the vapor pressure for the available chemicals. A regression line was fit to the points and is shown. The equation of this line is

$$
\frac{k_{s} / k_{d}}{d_{w}}=10^{2.93-0.31 \log V P} ; \quad R^{2}=0.85
$$

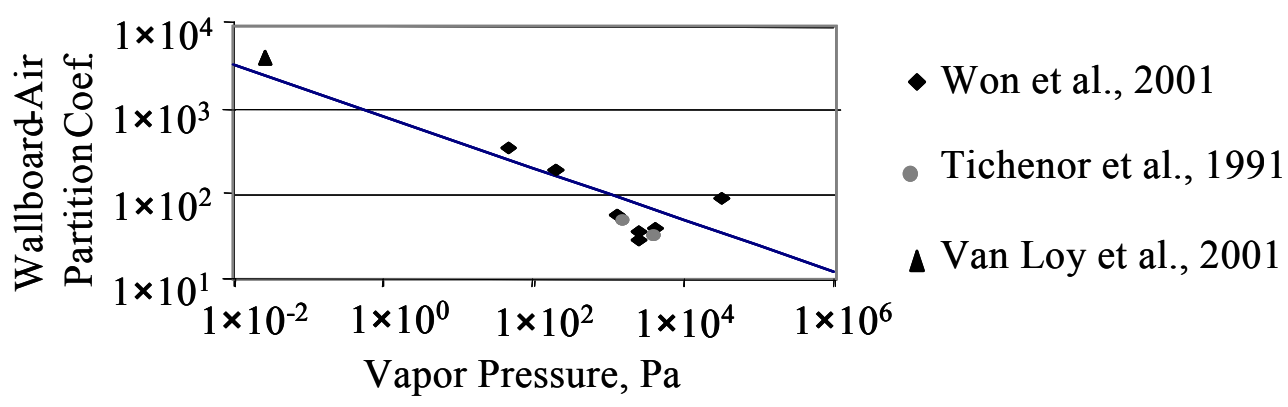

Figures 2. Surface partition coefficient versus vapor pressure from multiple experiments.

In addition to partition coefficients, we need experimental data to determine mass transfer rate coefficients from the air to the surface. Based on standard models, mass transfer is driven by the fugacity difference between air and the surface, and is controlled by a transfer resistance at this interface. On the air side of the interface, the resistance to transfer is proportional to the thickness of the thin boundary layer of stagnant air on the surface. In Figure 3 we plot the mass transfer rate to the wallboard versus the vapor pressure from the available experimental data. There is a maximum transfer rate based on the resistance through the air boundary layer. Chemicals with higher vapor pressures exhibit an additional resistance to transfer from the wallboard side of the interface. A line was fit to the data to determine the mass transfer rate coefficient for the higher volatility compounds:

$$
Y=10^{-1.63-0.41 \log V P} ; \quad R^{2}=0.67, \quad \text { Max. Value }=2.8 \times 10^{-2}
$$

where $\mathrm{Y}$ is the mass transfer coefficient $\left(\mathrm{mol} /\left(\mathrm{m}^{2} \mathrm{~Pa}-\mathrm{d}\right)\right)$. This is equal to a mass transfer coefficient in units of $\mathrm{m} / \mathrm{d}$ times the fugacity capacity of air, in units of $\mathrm{mol} / \mathrm{m}^{3} \mathrm{~Pa}$. 
We a use a similar approach to establish fugacity capacities and mass transfer rates for each residential surface. Because the quantity of available data varies by surface type, there are

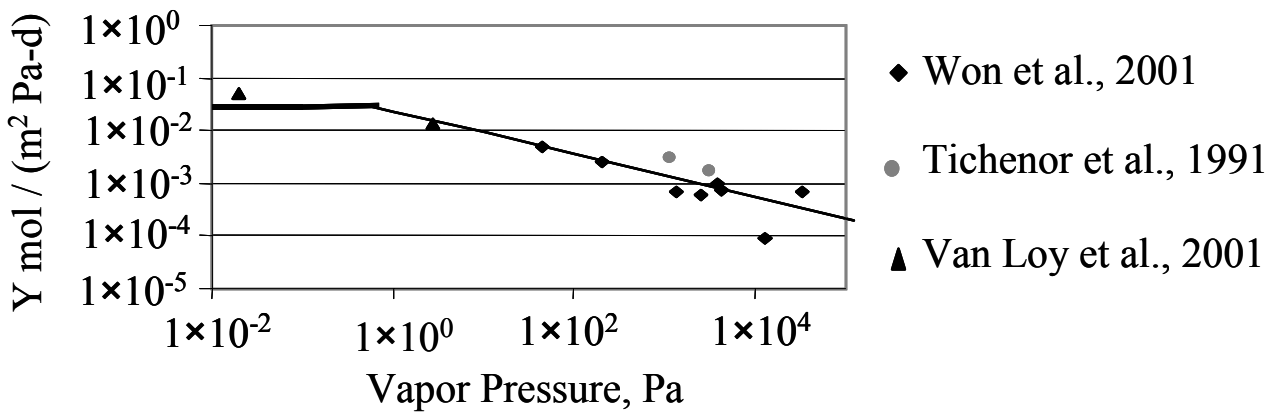

significant variations in the reliability of these estimates.

Figure 3. Transfer rate versus vapor pressure for partitioning to wallboard.

\section{DISCUSSION}

Limited availability of experimental data limits the complexity and reliability of our models. Although sufficient data are available to parameterize the holding capacity and transfer rates to and from some residential surfaces, more research is needed in this area to account for variations among chemicals, variations in surface types, and variations among homes. In particular, many of the partition coefficients have been measured for compounds with much higher volatility than the pesticides of current interest. In the current version of our model extrapolations from non-pesticide data are used to determine the partitioning into various surfaces. Controlled experiments with pesticides need to be completed to confirm partition coefficients and transfer rates for pesticides and other low-volatility compounds.

More household-specific measurements are needed on degradation rates and transfer rates. Most available data on degradation rates are from the outdoors. Many compounds that are degraded by photodegradation persist much longer indoors. However, the degradation rates in the indoor air and on indoor surfaces have not been quantified for the pesticides of concern.

Rates of transfer from surfaces to air following a crack and crevice application need to be better quantified through controlled experiments. A preliminary sensitivity analysis on the model indicated that uncertainty in this parameter leads to much uncertainty in model results.

\section{CONCLUSIONS}

A better understanding of pesticide fate and transport in the home is needed to quantify exposure to pesticides used in the home. A general framework for modeling fate and transport indoors is presented. The main challenge for further model development is to obtain more reliable estimates of the value ranges of partitioning, degradation, and transfer rates of pesticides on a variety of household surfaces.

\section{ACKNOWLEDGEMENTS}

This work was supported in part by the US Environmental Protection Agency National Exposure Research Laboratory through Interagency Agreement \# DW-988-38190-01-0 and carried out at Lawrence Berkeley National Laboratory through the US Department of Energy under Contract Grant No. DE-AC03-76SF00098. Dr. Bennett also acknowledges general support of her research under Kresge Center for Environmental Health, NIEHS ES000002. The authors also thank RL Corsi and WW Nazaroff for helpful input. 


\section{REFERENCES}

Byrne, S. L., B. A. Shurdut and D. G. Saunders (1998). "Potential chlorpyrifos exposure to residents following standard crack and crevice treatment." Environmental Health Perspectives V106(N11): 725-731.

Cox, S. S., D. Y. Zhao and J. C. Little (2001). "Measuring partition and diffusion coefficients for volatile organic compounds in vinyl flooring." Atmospheric Environment V35(N22): 3823-3830.

Diamond, M. L., S. E. Gingrich, K. Fertuck, et al. (2000). "Evidence for organic film on an impervious urban surface: Characterization and potential teratogenic effects." Environmental Science \& Technology V34(N14): 2900-2908.

Gordon, S., P. Callahan, M. Nishioka, et al. (1999). "Residential environmental measures in the National Human Exposure Assessment Survey (NHEXAS) pilot study in Arizona: preliminary results for pesticides and VOCs." Journal of Exposure Analysis and Environmental Epidemiology 9: 456-470.

Gurunathan, S., M. Robson, N. Freeman, et al. (1998). "Accumulation of Chlorpyrifos on Residential Surfaces and Toys Accessible to Children." Environmental Health Perspectives 106(1): 9-16.

Jorgensen, R. B., T. H. Dokka and O. Bjorseth (2000). "Introduction of a sink-diffusion model to describe the interaction between volatile organic compounds (VOCs) and material surfaces." Indoor Air-International Journal of Indoor Air Quality and Climate V10(N1): 27-38.

Leidy, R., C. Wright and H. J. Dupree (1993). Exposure Levels to Indoor Pesticides. Pesticides in Urban Environments. K. Racke and A. Leslie. Washington, DC, American Chemical Society.

Lewis, R. G., C. R. Fortune, F. T. Blanchard, et al. (2001). "Movement and deposition of two organophosphorus pesticides within a residence after interior and exterior applications." Journal of the Air \& Waste Management Association V51(N3): 339-351.

Mackay, D. (1991). Multimedia Environmental Models, the Fugacity Approach. Chelsea, MI, Lewis Publishers.

Mackay, D. and S. Paterson (1983). "Fugacity Models of Indoor Exposure to Volatile Chemicals." Chemoshere 12: 143-154.

Matoba, Y., J. Yoshimur, J. Ohnishi, et al. (1998). "Development of the simulation Model InPest for Prediction of the Indoor Behavior of Pesticides." J. Air \& Waste Manage. Assoc. 48: 969-978.

Neretnieks, I., J. Christiansson, L. Romero, et al. (1993). "Modelling of Emission and Reemission of Volatile Organic Compounds from Building Materials with Indoor Air Applications." Indoor Air 3: 2-11.

Tichenor, B., Z. Guo, J. Dunn, et al. (1991). "The Interaction of Vapour Phase Organic Compounds with Indoor Sinks." Indoor Air 1: 23-35.

Van Loy, M. D., W. J. Riley, J. M. Daisey, et al. (2001). "Dynamic behavior of semivolatile organic compounds in indoor air. 2. Nicotine and phenanthrene with carpet and wallboard." Environmental Science \& Technology V35(N3): 560-567.

Won, D., R. L. Corsi and M. Rynes (2001). "Sorptive interactions between VOCs and indoor materials." Indoor Air-International Journal of Indoor Air Quality and Climate V11(N4): 246-256.

Zartarian, V. G., H. Ozkaynak, J. M. Burke, et al. (2000). "A modeling framework for estimating children's residential exposure and dose to chlorpyrifos via dermal residue contact and nondietary ingestion." Environmental Health Perspectives V108(N6): 505514. 